\title{
Empirical Modeling and Simulation of Temporal Based Adaptive Mobility Model for MANET
}

\author{
Gurusamy Prema ${ }^{1,3}$, Chandrabose Aravindan ${ }^{2}$, Kirthivasan Kannan ${ }^{1}$, Rathinam Maheswaran ${ }^{3}$ \\ ${ }^{1}$ SASTRA University, Thanjavur, India \\ ${ }^{2}$ SSN College of Engineering, Chennai, India \\ ${ }^{3}$ Mepco Schlenk Engineering College, Sivakasi, India \\ E-mail: \{gprema, rmahes\}@mepcoeng.ac.in, aravindanc@ssn.edu.in, kkannan@maths.sastra.edu \\ Received December 14, 2010; revised February 20, 2011; accepted February 28, 2011
}

\begin{abstract}
Mobile Ad Hoc network (MANET) is a collection of wireless mobile nodes forming a temporary network without the aid of any established infrastructure. To conduct meaningful performance analysis of MANETs, it is essential that the simulation of mobility model should reflect the realistic mobility pattern of mobile nodes i.e. placement of mobile nodes at different intervals of time. The formation of spontaneous network depends heavily on the movement of different nodes in a particular practical scenario. This research focuses on the modeling and simulation of a temporal Adaptive Mobility Model which can be adapted to any dynamic practical scenario. The mobility in the realistic environment is simulated based on a Probability Transition Matrix named as Personal Behavior Model (PBM) and validated for a practical Health Care Environment. The formation of MANET is assumed to be based on the movement of the patient i.e. mobile nodes in the health care environment. Patients waiting in front of each service point for different time intervals are taken as results and compared with the actual data.
\end{abstract}

Keywords: MANET, Mobility Model, Probability Transition Matrix, System Dynamics

\section{Introduction}

A Mobile Ad Hoc Network (MANET) is a spontaneous, self-organizing network of mobile computing devices, having no fixed infrastructure or administrative support, where each mobile node also acts as a router of network packets. The primary function of the MANET is to facilitate communication between its constituent nodes. So, each node on the network may be either sender or receiver of packets, and all nodes works as router as discussed in [1]. MANET's performance is validated by simulation with the help of mobility models that describe and dictate the mobility pattern of the mobile nodes that take part in forming MANET. Mobility model mimic the movement of mobile nodes in MANET simulation and is a representation of a certain real or abstract world that contains moving entities. The mobility model describes the movement pattern of mobile user and how their location, change over time. The movement pattern of mobile nodes plays an important role in the performance analysis of wireless networks $[2,3]$. The mobility of the nodes affects the number of average connected paths, which in turn affect the performance of the routing algorithm [4]. In [5], S.Ray discussed some of the mobility models are realistic and they reflect as close as possible those real world scenarios.

Due to the dynamic nature of users in a MANET, a key challenge in the evaluation of mobility pattern is to conduct the performance analysis, with realistic mobility models that accurately reflect the mobile users' movement [6]. In their work, S.Gowrishankar et al. discussed about MANET environment, where mobile nodes are free to join or leave the network at any point of time, resulting in a highly dynamic network environment compare to wired network [7]. The advantage and importance of simulation of mobility model for MANET is discussed in [8]. Several mobility models have been developed for MANET simulations and mostly they are unrealistic mobility models. In the unrealistic mobility model, the node can move towards a randomly chosen destination with a randomly chosen speed and direction [9]. They are not suitable for real environment. In realistic mobility models, the node can move towards proper destination in- 
stead of randomly choosing the destination. An adaptive mobility model is developed in this work can be used to simulate various practical environments $[10,11]$. The dynamic MANET formulation in any environment [12] with its realistic movement of nodes at different time intervals can also be simulated by using the developed adaptive model.

The developed model is validated for a Health Care Environment having five Service Points/ Attraction Points and working from 9 am to $10 \mathrm{pm}$ with a maximum average of 106 patients per day by conducting a survey and observing the patients movement [13,14].

The paper is organized as follows: Development of Temporal Adaptive Mobility Model is discussed in Section 2 and Health care environment for which the model is developed is described in Section 3. Numerical example showing the simulation setup is given in Section 4. Simulation results are explained in Section 5. Finally Conclusion is given in Section 6.

\section{Development of Temporal Adaptive Mobility Model}

The proposed Temporal Adaptive Mobility Model is developed based on the following assumptions:

1) Nodes which are entering into the environment are assumed to carry a mobile device capable of forming MANET [9].

2) Each node has its own RF transmission range of coverage as per IEEE 802.11b standards [15].

3) A node may visit an attraction point only once to complete all the services performed at that attraction point.

When a mobile node enters into the region where other nodes transmission range overlaps with its own transmission range, the connectivity is established between the nodes and the MANET structure is formed [16]. The block diagram of the proposed temporal adaptive mobility model is given as shown in Figure 1.

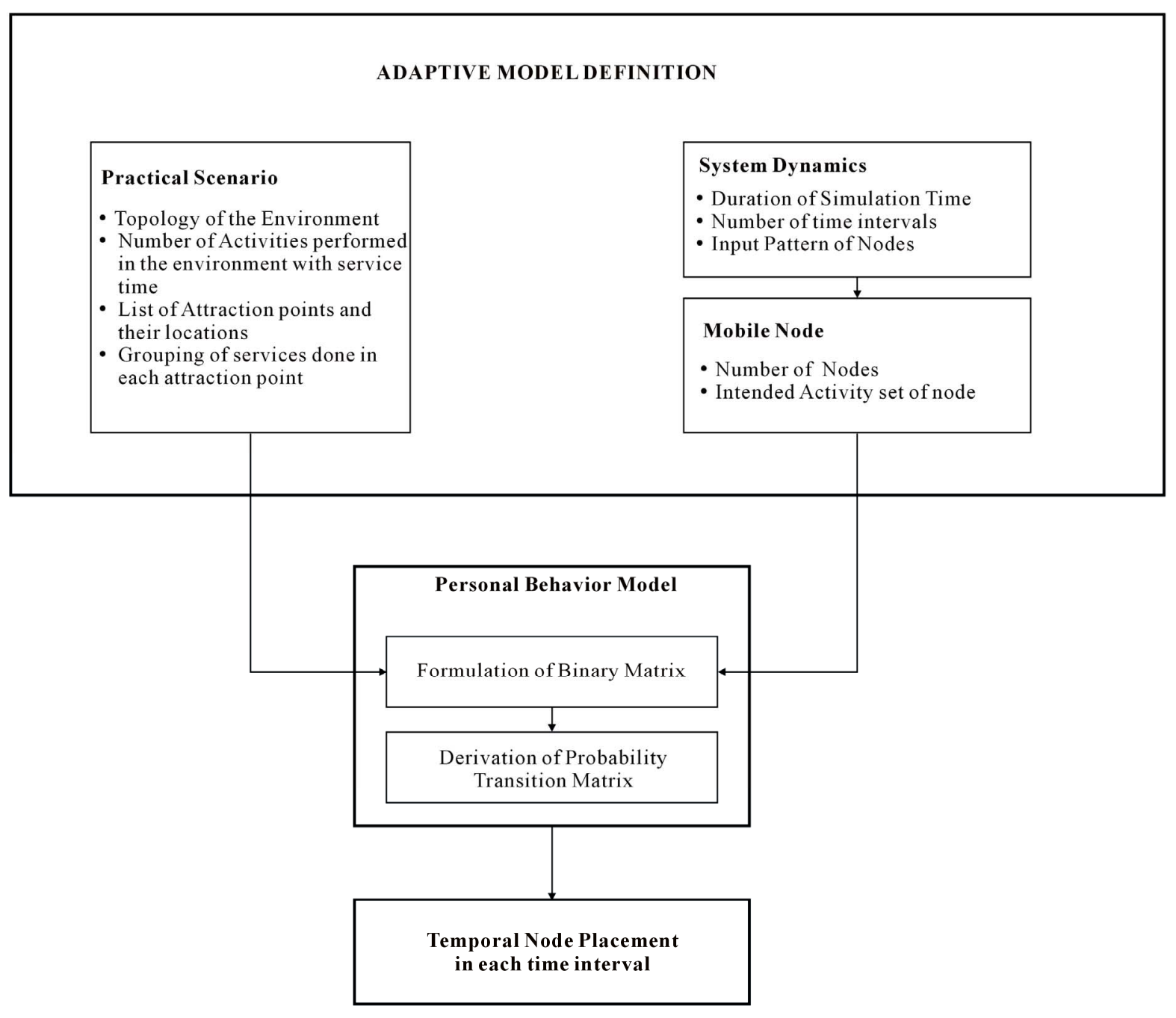

Figure 1. Adaptive temporal mobility model. 
The temporal adaptive mobility model is defined by the various inputs viz. 1) System dynamics based inputs, 2) Practical scenario based inputs and 3) Mobile node based inputs and they are used to formulate a Personal Behavior Model (PBM) which is a probability transition matrix and temporal node placement in different time interval to form spontaneous dynamic MANET within the environment.

\subsection{Temporal Adaptive Model Definition}

The temporal adaptive model suitable for any practical environment is defined by the following three inputs.

1) System dynamics based inputs.

2) Practical scenario based inputs.

3) Mobile node based inputs.

\subsubsection{System Dynamics Based Input}

The system dynamics of the adaptive mobility model is governed by two dynamic inputs namely 1) Duration of Simulation which is the time for which the environment is simulated and 2) Number of time intervals. The input pattern of nodes may follow a Probability Distribution Function over the simulation time. The total simulation period is the total period of observation in which the environment is simulated and duration of time interval can be calculated according to the number of time interval. At the start of each time interval the personal behavior model is updated.

\subsubsection{Practical Scenario Based Inputs}

The practical scenario based inputs [17] are environment specific inputs that specify the simulation environment according to the practical scenario. This consists of topology of the scenario which indicates the dimensions of the environment; various activities performed in the environment are listed out with their service time and given as total number of activities. Services are grouped and performed in particular service points. Various service points are listed out and given as inputs to the model with their locations.

\subsubsection{Mobile Node Based Inputs}

Any entity needing services from the practical scenario can be taken as nodes. These entities will enter into the practical scenario with an intended set of activities named as activity set and fulfilling their activities by the services performed at different service points and leave the system. So, any entity entering into the system has to move within the dimensions of environment and create individual mobility pattern within the environment during the simulation time. These will be given as mobile node based inputs. It is assumed that each entity is hav- ing a mobile device to formulate MANET at different time instances.

\subsection{Personal Behavior Model (PBM)}

Personal behavior model represents the intended real movement pattern of individual nodes in the environment based on the activities to be performed in the different attraction points. PBM is formulated by means of a binary matrix formed based upon the practical scenario and node based inputs at a particular time interval.

\subsubsection{Formulation of Binary Matrix}

Binary matrix is represented as a $M \times N$ matrix where $M$ is the number of nodes entering at a particular time interval and $N$ is the number of activities performed in the environment as shown in Table $\mathbf{1 .}$

The Binary matrix is formulated as follows: Elements $C_{i j}$ in the matrix represent whether $i^{\text {th }}$ Node performs $j^{\text {th }}$ activity or not. $C_{i j}$ is assigned as 'one'; if the $i^{\text {th }}$ node performs $j^{\text {th }}$ activity. Else $C_{i j}$ is assigned as 'zero'; i.e., the $i^{\text {th }}$ node does not perform $j^{\text {th }}$ activity.

For example, four nodes, $C_{1}, C_{2}, C_{3}$ and $C_{4}$ are entering into the environment with four different activity sets $A S_{1}, A S_{2}, A S_{3}$ and $A S_{4}$ respectively at a particular time interval. $C_{1}$ is entering with an activity set consists of activities $A_{1} \& A_{5} ; C_{2}$ is entering with an activity set consists of activities $A_{2} \& A_{3} ; C_{3}$ is entering with an activity set consists of activities $A_{1}, A_{4} \& A_{5}$ and $C_{4}$ is entering with an activity set consists of activity $A_{2}$. The corresponding entries in binary matrix are shown in the Table 2.

The binary matrix given in Table 2 will be the input for the derivation of PBM.

\subsubsection{Derivation of Personal Behavior Model}

Personal behavior model is a Probability Transition Matrix,

Table 1. Binary matrix.

\begin{tabular}{lllllllll}
\hline $\mathrm{N}$ & \multicolumn{8}{c}{ ACTIVITIES } \\
$\mathrm{O}$ & $C_{\mathrm{i}} / A_{\mathrm{j}}$ & $A_{1}$ & $A_{2}$ & $A_{3}$ & $A_{4}$ & $A_{5}$ & $\ldots$ & $A_{N}$ \\
$\mathrm{D}$ & $C_{1}$ & $C_{i j}$ & & & & & & \\
$\mathrm{E}$ & $\mathrm{S}$ & $C_{1}$ & & & & & & \\
$\mathrm{~S}$
\end{tabular}

Table 2. Formulation of binary matrix.

\begin{tabular}{lllllll}
\hline & \multicolumn{5}{c}{ ACTIVITIES } \\
\cline { 2 - 6 } $\mathrm{N}$ & $C_{i} / A_{j}$ & $A_{1}$ & $A_{2}$ & $A_{3}$ & $A_{4}$ & $A_{5}$ \\
$\mathrm{O}$ & $C_{1}$ & 1 & 0 & 0 & 0 & 1 \\
$\mathrm{D}$ & $C_{2}$ & 0 & 1 & 1 & 0 & 0 \\
$\mathrm{E}$ & $C_{2}$ & 1 & 0 & 0 & 1 & 1 \\
$\mathrm{~S}$ & $C_{3}$ & 0 & 1 & 0 & 0 & 0 \\
\hline
\end{tabular}


which indicates the probable movement of the nodes to fulfill the intended activities towards various service points in the environment. The matrix is having dynamic rows based upon the nodes available in the system other than waiting in front of the attraction points and number of attraction points as columns and updated at the starting of each time interval. This behavior model is used to denote the Probability of the nodes moving towards each attraction point. The Personal behavior model is derived as follows: Let $T_{i}$ be the total number of activities in the intended activity set of the $i^{\text {th }}$ node and $T_{i k}$ be the number of activities serviced in $k^{\text {th }}$ attraction point for $i^{\text {th }}$ node. Probability $P\left(C_{i}, A P_{k}\right)$ represents the probability of $i^{\text {th }}$ node in the system intended to move to the $k^{\text {th }}$ attraction point and given by the Equation (1).

$$
P\left(C_{i}, A P_{k}\right)=\frac{T_{i k}}{T_{i}}
$$

For a particular time interval, having the binary matrix as shown in Table 2, the PBM is derived as shown in Table 3.

Consider a practical environment, where there are three attraction points located and activities $A_{1}$ and $A_{2}$ are performed in $A P_{1}$, activities $A_{3}$ and $A_{5}$ are performed in $A P_{2}$ and activity $A_{4}$ is performed in $A P_{3}$. For the binary matrix shown in Table 3 , the entries in the PBM are as shown in Table 4.

After completion of all the activities, a node will move to next attraction point if it is having activity to do and after completing all the intended activities a node will leave the system.

Table 3. Formulation of personal behavior model.

\begin{tabular}{cccccc}
\hline \multicolumn{5}{c}{ Attraction Points } \\
\cline { 2 - 5 } $\mathrm{N}$ & $C_{i} / A P_{k}$ & $A P_{1}$ & $A P_{3}$ & $\ldots$ & $A P_{\mathrm{P}}$ \\
$\mathrm{O}$ & $C_{1}$ & & & & \\
$\mathrm{D}$ & $\vdots$ & $P\left(C_{i}, A P_{k}\right)$ & & \\
$\mathrm{E}$ & $C_{\mathrm{N}}$ & & \\
$\mathrm{S}$ & & & \\
\hline
\end{tabular}

Table 4. Personal behavior model.

\begin{tabular}{lllll}
\hline & \multicolumn{5}{c}{ Attraction Points } \\
\cline { 2 - 5 } $\mathrm{N}$ & $C_{\mathrm{i}} / A P_{k}$ & $A P_{1}$ & $A P_{2}$ & $A P_{3}$ \\
$\mathrm{O}$ & $C_{1}$ & 0.5 & 0.5 & 0 \\
$\mathrm{D}$ & $C_{2}$ & 0 & 1 & 0 \\
$\mathrm{E}$ & $C_{2}$ & 0.33 & 0.33 \\
$\mathrm{~S}$ & $C_{3}$ & 1 & 0 & 0 \\
\hline
\end{tabular}

\subsection{Temporal Node Placement}

For a given simulation time, different nodes are entering into the system and move according to their intended activity set. For any discrete time interval of the simulation, the nodes will be distributed over the entire dimensions of the environment. This distribution is purely based upon purpose of entering the system and leaving the system after the services are completed. So, the connectivity of the nodes will be spontaneous and it will form a self organizing network i.e. MANET. So, the node placement during the entire simulation time is taken as the mobility pattern of the system to formulate the MANET structure.

\section{Health Care Environment}

The Temporal adaptive mobility model developed is tested for a practical health care environment, where 31 different activities are performed at 5 different service points namely, Doctor's Room, Injection/Dressing Room, Pharmacy, Laboratory and Physiotherapy centre and is working from 9 a.m to 10 p.m. After making a pilot survey for a period of one month, it is observed that a maximum average of 106 patients is entering into the system to avail different services.

Health care environment is defined by having the system dynamic based inputs, the total simulation period 780 minutes is divided into 26 numbers of equal intervals, each of 30 minutes duration. First interval is from 9.00 am to $9.30 \mathrm{am}$, second interval is from 9.30 am to 10.00 am, etc. up to $26^{\text {th }}$ interval is from $9.30 \mathrm{pm}$ to $10 \mathrm{pm}$. It is assumed that Personal Behavior Matrix is updated at the start of each time interval. For the first time interval it is updated at 9.00 am, second time at 9.30 am etc. The Arrival pattern of the nodes into the system may follow a probability distribution and observed during the different time intervals is plotted as shown in Figure 2.

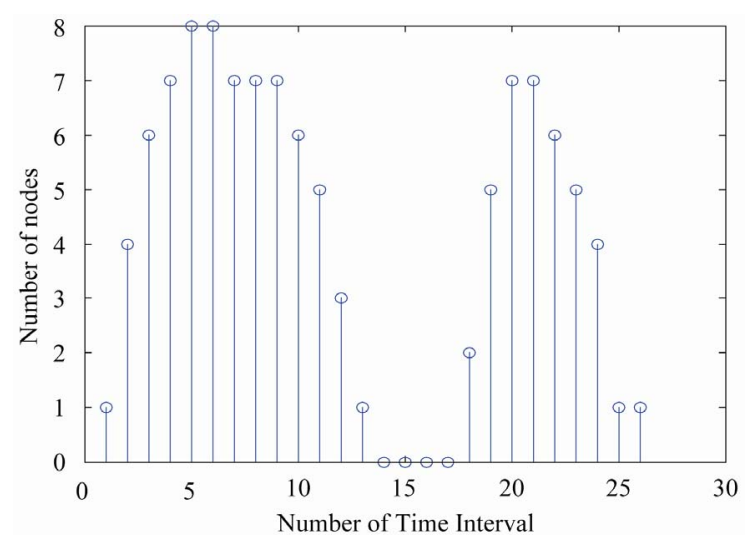

Figure 2. Number of nodes entered into the system in each time interval. 
Practical scenario based inputs showing area of the environment having the dimension $25 \mathrm{~m}$ (Length) and $16.5 \mathrm{~m}$ (width) and locations of service points are given in Figure 3.

15 different significant activities are selected by neglecting the rarely occurring activities performed in the environment with their service time are listed in Table 5.

The activities are grouped and serviced in the five different service points as shown in Table 6.

Node based inputs are the number of nodes entering into the environment for a particular time interval and the arrival pattern. For any interval of time, activity set is assigned to each node randomly following the Probability Distribution. Nodes which are entering will be assigned with any one of the intended activity set as shown in Table 7.

The formulation of binary matrix and derivation of PBM are explained using a numerical example.

\section{Numerical Example}

The development of the PBM for the above defined Health care environment explained in the previous section is illustrated as follows:

\subsection{System Dynamic Based Inputs}

Simulation time: 780 minutes

Number of time interval: 26

Duration: 30 minutes

\subsection{Practical Scenario Based Inputs}

Area of the environment: $25 \mathrm{~m} * 16.5 \mathrm{~m}$

Number of Activities performed: 15

Table 5. The list of activities and their service time.

\begin{tabular}{llll}
\hline $\begin{array}{c}\text { Activity } \\
\text { Set }\end{array}$ & \multicolumn{1}{c}{$\begin{array}{c}\text { List of } \\
\text { Activities }\end{array}$} & \multicolumn{1}{c}{$\begin{array}{c}\text { Activity } \\
\text { Set }\end{array}$} & \multicolumn{1}{c}{ List of activities } \\
\hline$A S_{1}$ & $A_{1} \& A_{4}$ & $A S_{18}$ & $A_{1}, A_{2}, A_{4}, A_{7} \& A_{10}$ \\
$A S_{2}$ & $A_{1}, A_{2} \& A_{4}$ & $A S_{19}$ & $A_{1} \& A_{3}$ \\
$A S_{3}$ & $A_{1}$ & $A S_{20}$ & $A_{1} \& A_{6}$ \\
$A S_{4}$ & $A_{1} \& A_{2}$ & $A S_{21}$ & $A_{1}, A_{4} \& A_{11}$ \\
$A S_{5}$ & $A_{1}, A_{4} \& A_{7}$ & $A S_{22}$ & $A_{1}, A_{2}, A_{4}, A_{7} \& A_{12}$ \\
$A S_{6}$ & $A_{1} \& A_{7}$ & $A S_{23}$ & $A_{3}$ \\
$A S_{7}$ & $A_{1}, A_{4} \& A_{13}$ & $A S_{24}$ & $A_{1}, A_{4} \& A_{8}$ \\
$A S_{8}$ & $A_{1}, A_{2}, A_{4} \& A_{7}$ & $A S_{25}$ & $A_{1}, A_{4} \& A_{10}$ \\
$A S_{9}$ & $A_{1}, A_{2} \& A_{7}$ & $A S_{26}$ & $A_{1}, A_{12} \& A_{13}$ \\
$A S_{10}$ & $A_{5}$ & $A S_{27}$ & $A_{1}, A_{2}, A_{3} \& A_{4}$ \\
$A S_{11}$ & $A_{1}, A_{4} \& A_{12}$ & $A S_{28}$ & $A_{1}, A_{2}, A_{4} \& A_{8}$ \\
$A S_{12}$ & $A_{1}, A_{2}, A_{4} \& A_{13}$ & $A S_{29}$ & $A_{1}, A_{7} \& A_{12}$ \\
$A S_{13}$ & $A_{1} \& A_{13}$ & $A S_{30}$ & $A 1, A_{4} \& A_{9}$ \\
$A S_{14}$ & $A_{1} \& A_{12}$ & $A S_{31}$ & $A_{1}, A_{4} \& A_{14}$ \\
$A S_{15}$ & $A_{1}, A_{4}, A_{7} \& A_{10}$ & $A S_{32}$ & $A_{1}, A_{4} \& A_{15}$ \\
$A S_{16}$ & $A_{1}, A_{3} \& A_{4}$ & $A S_{33}$ & $A_{1}, A_{7} \& A_{10}$ \\
$A S_{17}$ & $A_{1}, A_{2} \& A_{13}$ & $A S_{34}$ & $A_{1}, A_{2}, A_{4} \& A_{12}$ \\
\hline & & & \\
\end{tabular}

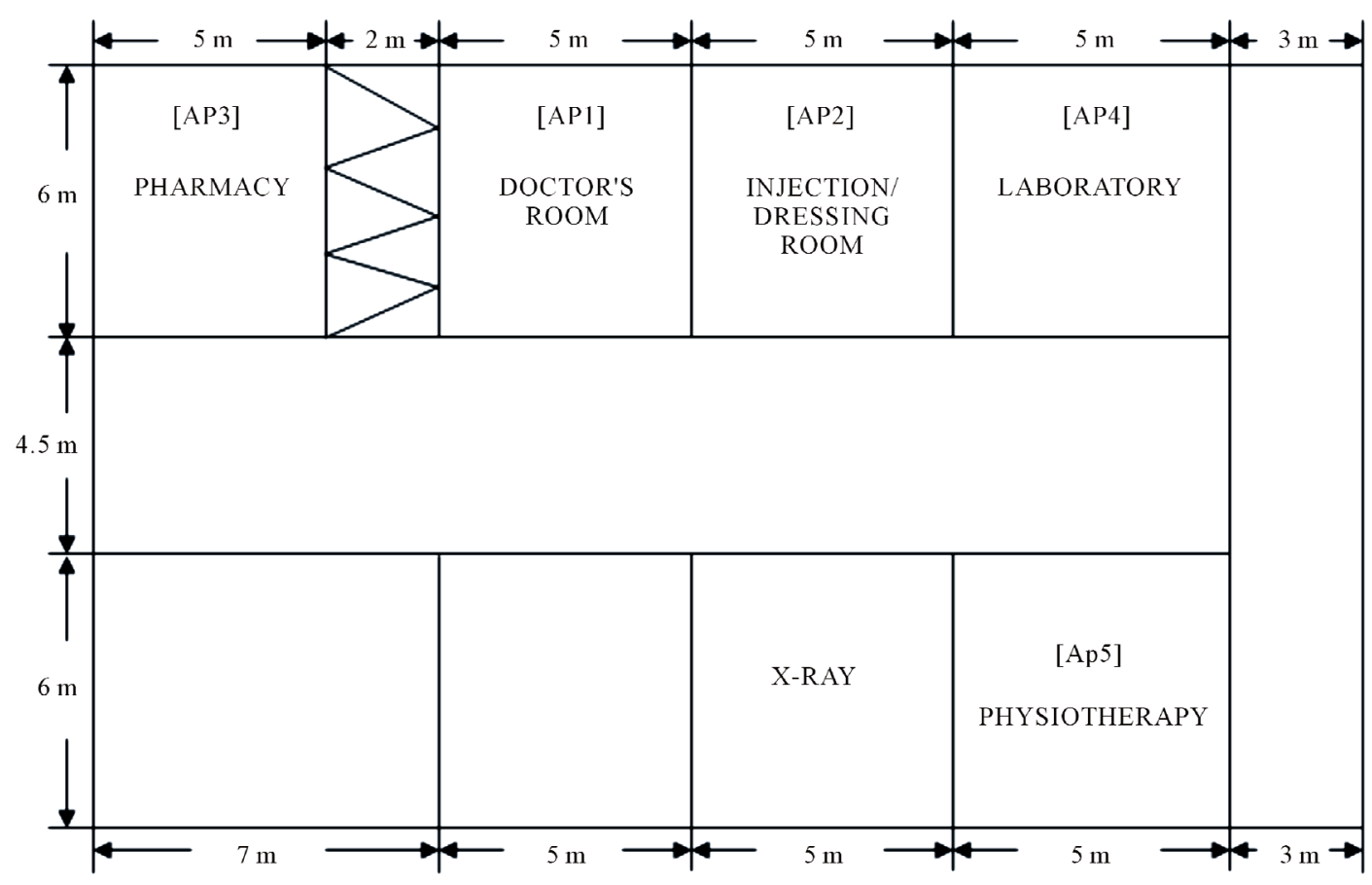

Figure 3. Topology of the environment showing the locations of Service points. 
Table 6. Attraction point and activities performed in them.

\begin{tabular}{cll}
\hline $\begin{array}{c}\text { Attraction } \\
\text { Point }\end{array}$ & \multicolumn{1}{c}{ Name } & \multicolumn{1}{c}{ Activities } \\
\hline$A P_{1}$ & $\begin{array}{l}\text { Doctor's Room } \\
\text { Injection/Dressing }\end{array}$ & $A_{1}$ \\
$A P_{2}$ & $\begin{array}{l}\text { Room } \& A_{3} \\
\end{array}$ & Pharmacy \\
$A P_{3}$ & $A_{4} \& A_{5}$ \\
$A P_{4}$ & Laboratory & $\begin{array}{l}A_{6}, A_{7}, A_{8}, A_{9}, A_{10}, A_{11}, A_{12} \\
\& A_{13}\end{array}$ \\
& Physiotherapy & $A_{14} \& A_{15}$ \\
\hline
\end{tabular}

Table 7. Activity set.

\begin{tabular}{clll}
\hline $\begin{array}{c}\text { Activity } \\
\text { Set }\end{array}$ & \multicolumn{1}{c}{$\begin{array}{c}\text { List of } \\
\text { Activities }\end{array}$} & $\begin{array}{c}\text { Activity } \\
\text { Set }\end{array}$ & \multicolumn{1}{c}{ List of Activities } \\
\hline$A S_{1}$ & $A_{1} \& A_{4}$ & $A S_{18}$ & $A_{1}, A_{2}, A_{4}, A_{7} \& A_{10}$ \\
$A S_{2}$ & $A_{1}, A_{2} \& A_{4}$ & $A S_{19}$ & $A_{1} \& A_{3}$ \\
$A S_{3}$ & $A_{1}$ & $A S_{20}$ & $A_{1} \& A_{6}$ \\
$A S_{4}$ & $A_{1} \& A_{2}$ & $A S_{21}$ & $A_{1}, A_{4} \& A_{11}$ \\
$A S_{5}$ & $A_{1}, A_{4} \& A_{7}$ & $A S_{22}$ & $A_{1}, A_{2}, A_{4}, A_{7} \& A_{12}$ \\
$A S_{6}$ & $A_{1} \& A_{7}$ & $A S_{23}$ & $A_{3}$ \\
$A S_{7}$ & $A_{1}, A_{4} \& A_{13}$ & $A S_{24}$ & $A_{1}, A_{4} \& A_{8}$ \\
$A S_{8}$ & $A_{1}, A_{2}, A_{4} \& A_{7}$ & $A S_{25}$ & $A_{1}, A_{4} \& A_{10}$ \\
$A S_{9}$ & $A_{1}, A_{2} \& A_{7}$ & $A S_{26}$ & $A_{1}, A_{12} \& A_{13}$ \\
$A S_{10}$ & $A_{5}$ & $A S_{27}$ & $A_{1}, A_{2}, A_{3} \& A_{4}$ \\
$A S_{11}$ & $A_{1}, A_{4} \& A_{12}$ & $A S_{28}$ & $A_{1}, A_{2}, A_{4} \& A_{8}$ \\
$A S_{12}$ & $A_{1}, A_{2}, A_{4} \& A_{13}$ & $A S_{29}$ & $A_{1}, A_{7} \& A_{12}$ \\
$A S_{13}$ & $A_{1} \& A_{13}$ & $A S_{30}$ & $A 1, A_{4} \& A_{9}$ \\
$A S_{14}$ & $A_{1} \& A_{12}$ & $A S_{31}$ & $A_{1}, A_{4} \& A_{14}$ \\
$A S_{15}$ & $A_{1}, A_{4}, A_{7} \& A_{10}$ & $A S_{32}$ & $A_{1}, A_{4} \& A_{15}$ \\
$A S_{16}$ & $A_{1}, A_{3} \& A_{4}$ & $A S_{33}$ & $A_{1}, A_{7} \& A_{10}$ \\
$A S_{17}$ & $A_{1}, A_{2} \& A_{13}$ & $A S_{34}$ & $A_{1}, A_{2}, A_{4} \& A_{12}$ \\
\hline & & &
\end{tabular}

Number of Attraction points: 5

No. of Activities performed in $A P_{1}: 1$

No. of Activities performed in $A P_{2}: 2$

No. of Activities performed in $A P_{3}: 2$

No. of Activities performed in $A P_{4}: 8$

No. of Activities performed in $A P_{5}: 2$

\subsection{Node Based Inputs at the First Time Interval $t_{1}$ From 9.00 Am to 9.30 Am}

The number of nodes entering in first time interval from $9.00 \mathrm{am}$ to $9.30 \mathrm{am}$ is one and the intended activity set assigned to this node among 34 activity set is $A S_{1}$, which consists of two activities $A_{1}$ and $A_{4}$. Binary matrix formulated at the start of first time interval is as shown in Table 8.
The node has to visit two attraction points namely, $A P_{1}$ to fulfill the activity $A_{1}$ and $A P_{3}$ to fulfill the activity $A_{4}$. The probability of visit to different attraction points are calculated by using equation (1). Therefore, $P\left(C_{1}, A P_{1}\right)$ $=0.5, P\left(C_{1}, A P_{2}\right)=0, P\left(C_{1}, A P_{3}\right)=0.5, P\left(C_{1}, A P_{4}\right)=0$ and $P\left(C_{1}, A P_{5}\right)=0$. Personal behavior matrix derived for the first time interval is given in Table 9 .

This PBM will be updated according to the entry of nodes in time interval 2, time interval 3 etc. For a middle interval, i.e. at the start of seventh time interval $t_{7}$, from $12 \mathrm{pm}$ to $12.30 \mathrm{pm}$, the number of nodes arrived during this interval are 7 (from $C_{35}$ to $C_{41}$ ). Up to the $6^{\text {th }}$ interval, 34 nodes entered into the environment. 8 Nodes fulfilled the services and left the system and at the start of $6^{\text {th }}$ interval, 26 nodes are present in the system at the start of the interval. At the end of $6^{\text {th }}$ interval, one node left the system. The corresponding Binary matrix formulated at the start of seventh time interval is shown in Table $\mathbf{1 0 .}$

Personal behavior matrix derived for this time interval is given in Table 11.

Table 8. Binary matrix formulated at first time interval.

\begin{tabular}{cccccccccccccccc}
\hline$C_{i} / A_{j}$ & $A_{1}$ & $A_{2}$ & $A_{3}$ & $A_{4}$ & $A_{5}$ & $A_{6}$ & $A_{7}$ & $A_{8}$ & $A_{9}$ & $A_{10}$ & $A_{11}$ & $A_{12}$ & $A_{13}$ & $A_{14}$ & $A_{15}$ \\
\hline $\mathrm{C}_{1}$ & 1 & 0 & 0 & 1 & 0 & 0 & 0 & 0 & 0 & 0 & 0 & 0 & 0 & 0 & 0 \\
\hline
\end{tabular}

Table 9. Personal behavior matrix derived atfirst time interval.

\begin{tabular}{cccccc}
\hline $\mathrm{C}_{\mathrm{i}} / \mathrm{AP}_{\mathrm{k}}$ & $\mathrm{AP}_{1}$ & $\mathrm{AP}_{2}$ & $\mathrm{AP}_{3}$ & $\mathrm{AP}_{4}$ & $\mathrm{AP}_{5}$ \\
\hline $\mathrm{C}_{1}$ & 0.5 & 0 & 0.5 & 0 & 0 \\
\hline
\end{tabular}

Table 10. Binary matrix formulated at seventh time interval.

\begin{tabular}{lllllllllllllllll}
\hline$C_{i} / A_{j}$ & $A_{1}$ & $A_{2}$ & $A_{3}$ & $A_{4}$ & $A_{5}$ & $A_{6}$ & $A_{7}$ & $A_{8}$ & $A_{9}$ & $A_{10}$ & $A_{11}$ & $A_{12}$ & $A_{13}$ & $A_{14}$ & $A_{15}$ \\
\hline$C_{35}$ & 1 & 0 & 0 & 0 & 0 & 0 & 0 & 0 & 0 & 0 & 0 & 0 & 0 & 0 & 0 \\
$C_{36}$ & 1 & 1 & 0 & 1 & 0 & 0 & 0 & 0 & 0 & 0 & 0 & 0 & 0 & 0 & 0 \\
$C_{37}$ & 1 & 0 & 0 & 1 & 0 & 0 & 0 & 0 & 0 & 0 & 0 & 0 & 0 & 0 & 0 \\
$C_{38}$ & 1 & 0 & 0 & 1 & 0 & 0 & 0 & 0 & 0 & 0 & 0 & 0 & 0 & 0 & 0 \\
$C_{39}$ & 1 & 0 & 0 & 1 & 0 & 0 & 0 & 0 & 0 & 0 & 0 & 0 & 0 & 0 & 0 \\
$C_{40}$ & 1 & 0 & 0 & 1 & 0 & 0 & 0 & 0 & 0 & 0 & 0 & 0 & 0 & 0 & 0 \\
$C_{41}$ & 1 & 0 & 0 & 1 & 0 & 0 & 0 & 0 & 0 & 0 & 0 & 0 & 0 & 0 & 0 \\
\hline
\end{tabular}

Table 11. Personal behavior matrix at time interval $\mathbf{t}_{7}$.

\begin{tabular}{llllll}
\hline$C_{i} / A P_{k}$ & $A P_{1}$ & $A P_{2}$ & $A P_{3}$ & $A P_{4}$ & $A P_{5}$ \\
\hline$C_{35}$ & 1 & 0 & 0 & 0 & 0 \\
$C_{36}$ & 0.333 & 0.333 & 0.333 & 0 & 0 \\
$C_{37}$ & 0.5 & 0 & 0.5 & 0 & 0 \\
$C_{38}$ & 0.5 & 0 & 0.5 & 0 & 0 \\
$C_{39}$ & 0.5 & 0 & 0.5 & 0 & 0 \\
$C_{40}$ & 0.5 & 0 & 0.5 & 0 & 0 \\
$C_{41}$ & 0.5 & 0 & 0.5 & 0 & 0 \\
\hline
\end{tabular}


For each time interval, if there is any entity waiting for service at attraction points, node has to join in the queue for their service. This placement of different nodes in the environment will create a dynamic MANET topology and connectivity among the nodes for the entire simulation period.

\section{Simulation Results}

The temporal adaptive model is defined and simulated with VC++ in a Pentium IV computer with 1 GB RAM working in Windows XP Professional O.S platform version 2. The arrival of nodes into the health care environment, their movement patterns i.e. the placement of nodes at different time interval is simulated. The results of simulation viz. mobile nodes placement in different attraction points for each time interval, number of nodes arrived, total number of nodes present in the system at the start of the interval, number of nodes which are waiting in front of APs at the start of the interval and the number of nodes left the system at the end of the interval are tabulated and shown in Table 12.

Table 12. Mobile nodes details in front of all APs in each time interval.

\begin{tabular}{|c|c|c|c|c|c|c|c|c|}
\hline \multirow{2}{*}{ Time Interval } & \multirow{2}{*}{$\begin{array}{c}\text { No. of nodes } \\
\text { arrived }\end{array}$} & \multirow{2}{*}{$\begin{array}{l}\text { Total no. of } \\
\text { nodes in the } \\
\text { system }\end{array}$} & \multicolumn{5}{|c|}{ No. of nodes in APs at the start of the interval } & \multirow{2}{*}{$\begin{array}{l}\text { No. of nodes left the } \\
\text { system at the end of } \\
\text { the interval }\end{array}$} \\
\hline & & & $\mathbf{A P}_{1}$ & $\mathbf{A P}_{2}$ & $\mathbf{A P}_{3}$ & $\mathbf{A P}_{4}$ & $\mathbf{A P}_{5}$ & \\
\hline 1 & 1 & 1 & 1 & - & - & - & - & 1 \\
\hline 2 & 4 & 4 & 2 & - & 2 & - & - & 1 \\
\hline 3 & 6 & 9 & 3 & 2 & 4 & - & - & 2 \\
\hline 4 & 7 & 14 & 5 & 3 & 6 & - & - & 1 \\
\hline 5 & 8 & 21 & 8 & 4 & 9 & - & - & 3 \\
\hline 6 & 8 & 26 & 10 & 5 & 11 & - & - & 1 \\
\hline 7 & 7 & 32 & 11 & 6 & 15 & - & - & 3 \\
\hline 8 & 7 & 36 & 13 & 5 & 17 & 1 & - & 3 \\
\hline 9 & 7 & 40 & 14 & 7 & 19 & - & - & 4 \\
\hline 10 & 6 & 42 & 15 & 7 & 20 & - & - & 1 \\
\hline 11 & 5 & 46 & 15 & 8 & 23 & - & - & 1 \\
\hline 12 & 3 & 48 & 15 & 6 & 27 & - & - & 3 \\
\hline 13 & 1 & 46 & 10 & 6 & 30 & - & - & 4 \\
\hline 14 & 0 & 42 & 5 & 4 & 33 & - & - & 2 \\
\hline 15 & 0 & 40 & - & 5 & 35 & - & - & 2 \\
\hline 16 & 0 & 38 & 1 & 3 & 34 & - & - & 2 \\
\hline 17 & 0 & 36 & - & 3 & 33 & - & - & 2 \\
\hline 18 & 2 & 36 & 2 & 1 & 33 & - & - & 2 \\
\hline 19 & 5 & 39 & 4 & 1 & 34 & - & - & 2 \\
\hline 20 & 7 & 44 & 5 & 2 & 36 & 1 & - & 3 \\
\hline 21 & 7 & 48 & 8 & 1 & 39 & - & - & 3 \\
\hline 22 & 6 & 51 & 11 & 2 & 38 & - & - & 2 \\
\hline 23 & 5 & 54 & 9 & 2 & 43 & - & - & 3 \\
\hline 24 & 4 & 55 & 9 & 1 & 45 & - & - & 3 \\
\hline 25 & 1 & 53 & 4 & 3 & 46 & - & - & 3 \\
\hline 26 & 1 & 51 & 1 & 1 & 49 & - & - & 2 \\
\hline
\end{tabular}


It is observed from the results that more number of mobile nodes is waiting for service at $A P_{1} \& A P_{2}$ than $A P_{3}, A P_{4} \& A P_{5}$ since in most of the activity sets, activities to be done at $A P_{3}, A P_{4} \& A P_{5}$ are rarely occurring when compared to other activities done in this particular simulated Health care environment. Therefore, nodes get services in these attraction points without waiting in the queue. It is also observed that size of the queue in front of $A P_{1}$, i.e. at Doctor's Room is having a peak from 12 pm to $3 \mathrm{pm}$ and around $7 \mathrm{pm}$. Number of nodes in $A P_{3}$, i.e. at Pharmacy is increasing up to last time interval since most of the patients has to visit Pharmacy in the health care environment. The number of nodes waiting in front of the various service points is shown in Figure 4.

From the Figure 4, it is observed that the nodes waiting in front of Laboratory and Physiotherapy centre are minimum. Maximum numbers of nodes are waiting in front of Doctors room and Pharmacy. It is also verified from the observed data that always Pharmacy is overcrowded. The number of nodes present in the system for various time intervals is given in Figure 5.

The simulation results showing nodes leaving pattern is shown in Figure 6.

In the simulation it is assumed that all the nodes will the system only after fulfilling the intended activities. Therefore the nodes present in the system have to wait in the queue for long time as the number of nodes entered as per the arrival pattern. But the real time data observed

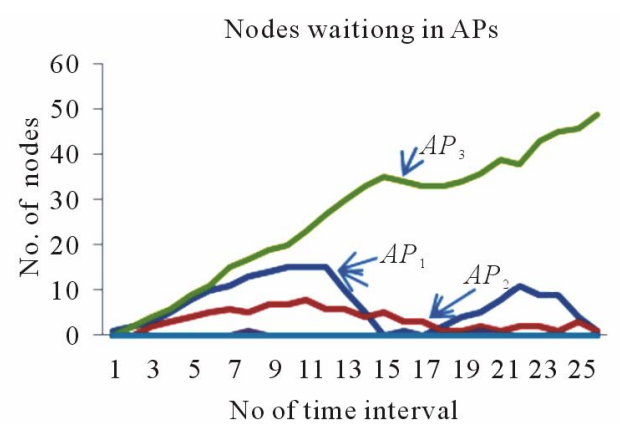

Figure 4. Nodes in attraction points at different time intervals.

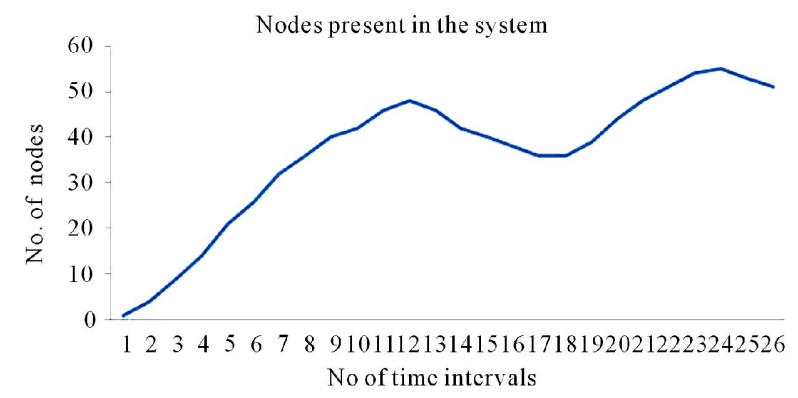

Figure 5. Nodes present in the system at different time intervals.

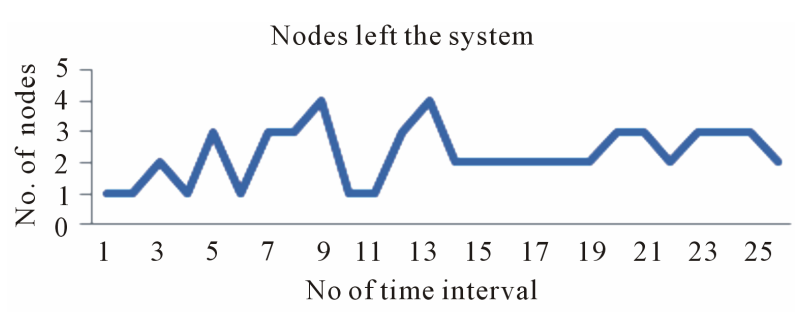

Figure 6. Nodes left the system at different time intervals.

in the environment during survey is having the leaving pattern of the nodes that some of the nodes left the system after consultation without getting medicine from the pharmacy.

\section{Conclusions}

A temporal adaptive mobility model is developed which can be used to simulate any practical scenario by giving the appropriate inputs. The model is simulated for a health care environment by assuming patients as nodes which moves towards various service points like Doctor's Room, Injection/Dressing Room, Pharmacy etc. and the mobility patterns are analyzed. The output of the simulated model reflects exactly the dynamic movement pattern of the mobile entities as observed in the environment. The simulated results are similar to the practical environment but the number of nodes queue in front of pharmacy is not correlated because of dejected leaving patients from the system.

\section{Acknowledgements}

The authors are thankful to the Management, Principal and Head of the Electronics and Communication Engineering Department, Mepco Schlenk Engineering College, Sivakasi, Tamilnadu, India, for giving the support to carry out this research work.

\section{References}

[1] M. Kim, D. Kotz and S. Kim, "Extracting a Mobility Model from Real User Traces,” Proceedings of the 25th Annual Joint Conference of the IEEE Computer and Communications Societies, Barcelona, 23-29 April 2006, pp. 1-13.

[2] M. McNett and G. M. Voelker, "Access and Mobility of Wireless PDA Users,” Mobile Computing Communications Review, Vol. 9, No. 2, 2005, pp. 40-55. doi:10.1145/1072989.1072995

[3] B. Divecha, A. Abraham, C. Grosan and S. Sanyal, "Impact of Node Mobility on MANET Routing Protocols Models," Journal of Digital Information Management, Vol. 5, No. 1, 2007, pp. 19-24.

[4] A. J. Pullin, "A Realistic Model for Evaluation of MA- 
NET," Proceedings of Innovation North Research \& Practice Conference, Leeds, 9-11 July 2007, pp. 1-15.

[5] S. Ray, "Realistic Mobility for MANET Simulation," Master's Thesis, The University of British Columbia, Vancouver, 2003.

[6] A. Jardosh, E. M. Belding-Royer, K. C. Almeroth and S. Suri, "Real World Environment Models for Mobile AdHoc Networks Evaluation," IEEE Journal on Selected Areas in Communications, Special Issue on Wireless Ad Hoc Networks, Vol. 23, No. 3, 2005, pp. 622-632.

[7] S. Gowrishankar, T. G. Basavaraju and S. Sarkar, "Effect of Random Mobility Models Pattern in Mobile Ad Hoc Networks,” International Journal of Computer Science and Network Security, Vol. 7, No. 6, 2007, pp. 160-164.

[8] P. Venkateswaran, R. Ghosh, A. Das, S. K. Sanyal and R. Nandi, "An Obstacle Based Realistic Ad-Hoc Mobility Model for Social Networks,” Journal of Networks, Vol. 1, No. 2, 2006, pp. 37-44.

[9] T. Camp, J. Boleng and V. Davies, "A Survey of Mobility Models for Ad Hoc Network Research," Wireless Communication and Mobile Computing: Special Issue on Mobile Ad Hoc Networking: Research, Trends and Applications, Vol. 2, No. 5 , 2002, pp. 483-502.

[10] A. J. Pullin and C. Pattinson, "A Realistic Battlefield Model for the Evaluation of MANET," Proceedings of 5th Annual Conference on Wireless on Demand Network Systems and Services, Garmisch Partenkirchen, 23-25 January 2008, Vol. 23, pp. 81-84. doi:10.1109/WONS.2008.4459359

[11] T. M. L. Flower, G. Prema, C. Aravindan, K. Kannan and
R. Maheswaran, “A Realistic Mobility Pattern for Adaptive MANET Simulation Environment," Proceedings of National Conference on Networks, Image \& Security, Kumarakoil, 14-15 March 2008, pp. 70-75.

[12] G. Lu, G. Manson and D. Belis, "Mobility Modeling in Mobile Ad Hoc Networks with Environment-Aware," Journal of Networks, Vol. 1, No. 1, 2006, pp. 54-63.

[13] D. Bhattacharjee, A. C. R. Shah, M. Shah and A. Helmy, "Empirical Modeling of Campus-Wide Pedestrian Mobility: Observations on the USC Campus,” Proceedings of IEEE Vehicular Technology Conference, Los Angeles, 2629 September 2004 Vol. 4, pp. 2887-2891.

[14] J. Kim and S. Bohacek, “A Survey-Based Mobility Model of People for Simulation of Urban Mesh Networks," Proceedings of Mesh Nets, Budapest, July 2005, pp. 1-11.

[15] D. Huang, "Unlinkability Measure for IEEE 802.11 Based MANETs,” IEEE Transactions on Wireless Communications, Vol. 7, No. 2, 2008, pp. 1025-1034. doi:10.1109/TWC.2008.060777

[16] M. A. Rajan, M. G. Chandra, L. C. Reddy and P. S. Hiremath, "Topological and Energy Analysis of K-Connected MANETs: A Semi-Analytical Approach,” International Journal of Computer Science and Network Security, Vol. 8, No. 2, 2008, pp. 199-207.

[17] P. Johansson, T. Larsson, N. Hedman, B. Mielczarek and M. Degermark, "Scenario Based Performance Analysis of Routing Protocols for Mobile Ad Hoc Networks," Proceedings of the 5th ACM Annual International Conference on Mobile Computing and Networking, Seattle, 1519 August 1999, pp. 195-206. 